\title{
2D magnetic MOFs with micron-lateral size by liquid exfoliation
}

\author{
Luis León-Alcaide, ${ }^{a}$ Javier López-Cabrelles, ${ }^{a}$ Guillermo Mínguez Espallargas ${ }^{a}$ and Eugenio \\ Coronado $^{a}$
}

The isolation in large amounts of high-quality flakes of 2D MOFs remains a challenge. In this work, we develop a liquid exfoliation procedure to obtain nanosheets for a whole family of Fe-based magnetic MOFs, MUV-1-X. High-quality crystalline layers with lateral sizes of $8 \mu \mathrm{m}$ and thicknesses of $4 \mathrm{~nm}$, which keep the structural integrity and magnetic properties, are obtained.

Since the discovery of graphene, other layered materials formed by one or few atomically-thin layers (aka. 2D materials) have been isolated. ${ }^{1,2}$ In this context, $2 \mathrm{D}$ MOFs are emerging as an important class of 2D materials of current interest, ${ }^{3}$ in different areas such as catalysis, ${ }^{4}$ membranes, ${ }^{5}$ energy, ${ }^{6}$ electronics, ${ }^{7}$ or magnetism. ${ }^{8}$ In magnetism for example, these 2D MOFs have shown to provide unique examples of magnetic layers which, in contrast to the $2 \mathrm{D}$ inorganic analogues, ${ }^{9}$ are chemically stable and more versatile from the point of view of their functionalization. ${ }^{10-12}$

Both top-down -involving the exfoliation of crystalline layered materials ${ }^{13}$ - and, to a less extent, bottom-up methodologies involving a direct synthesis of the 2D material from molecular precursors $^{14}$ - have been used to obtain 2D MOFs. Within the group of top-down methodologies, two different techniques have been developed to obtain nanosheets from the bulk, namely micromechanical ${ }^{15}$ and liquid exfoliation approaches. ${ }^{16}$ The quality of layers that can be obtained using these two approaches is very different. The micromechanical exfoliation, also known as the Scotch-tape method, allows to separate the layers without any interaction with solvents, thus affording very high-quality layers, which are necessary to explore the physics in the $2 \mathrm{D}$ limit. ${ }^{17}$ However, this dry approach is extremely difficult to apply to MOFs due to the fragility of the coordination compounds. ${ }^{10}$ In addition, it is not scalable as it only provides very small quantities of the 2D material, thus preventing its use in important applications such as membranes, ${ }^{18}$ sensors ${ }^{19}$ or catalysis. ${ }^{20}$ On the contrary, the quality of the layers obtained by liquid exfoliation is much lower since the sheets are typically damaged by the solvents used for their separation and stabilization. ${ }^{21}$ In addition, the size of the exfoliated layers is typically much smaller and it is very difficult to go down to the monolayer. However, this approach provides an attractive, simple and scalable method for the creation of ultrathin nanosheets from layered materials, which can be applied to MOFs. ${ }^{22}$ In view of these features, a challenging goal is to obtain 2D nanomaterials with micrometer-scale lateral dimensions and nanoscale longitudinal dimensions via liquid exfoliation, which would benefit from an easy processability while retaining the structural integrity.

Herein we demonstrate the production of nanosheets of good quality of 2D magnetic MOFs with lateral sizes of several microns using a liquid exfoliation approach. The compounds of interest are the so called MUV-1-X (MUV = Material of the University of Valencia), a family of layered coordination polymers of formula [Fe(bimX) $)_{2}$ ( $\mathrm{HbimX}=$ benzimidazole functionalized in the 5-position with $\mathrm{X}=\mathrm{H}, \mathrm{Cl}, \mathrm{Br}$, or $\mathrm{CH}_{3}$ ). Very recently we have shown that they can be exfoliated down to the monolayer using a dry micromechanical procedure. ${ }^{11}$ These crystalline materials are formed by tetrahedral $\mathrm{Fe}(\mathrm{II})$ centers linked via benzimidazolate bridges in order to form neutral layers of square Fe(II) networks separated by weak van der Waals interactions. From a magnetic point of view they behave in bulk as canted antiferromagnets ordering at $c a$. 19-20 $\mathrm{K}$.

MUV-1-X single crystals $\left(\mathrm{X}=\mathrm{H}, \mathrm{Cl}, \mathrm{Br}\right.$, or $\left.\mathrm{CH}_{3}\right)$ were prepared following the solvent-free methodology previously used by our group. ${ }^{11,23}$ Crystals of MUV-1-Cl were used as model compound of this family in order to assess the best conditions for the liquid exfoliation, analysing the effect of different parameters such as source of energy, solvent, time, temperature, concentration, and centrifugation. The exfoliation process is illustrated in Figure 1.

$2.5 \mathrm{mg}$ of MUV-1-Cl was initially added in $20 \mathrm{ml}$ of a given solvent, which was subsequently introduced both in a sonic bath and in the sonication tip to examine the impact caused by the source of sonication in the structure of the material. As has been previously established, ${ }^{22}$ the tip-assisted sonication can reach higher power than the bath-assisted sonication, resulting in the fragmentation onto smaller crystals with undefined morphologies. This is clearly evidenced in our case, where the sonication tip causes the disintegration of the material, whereas the sonication bath 

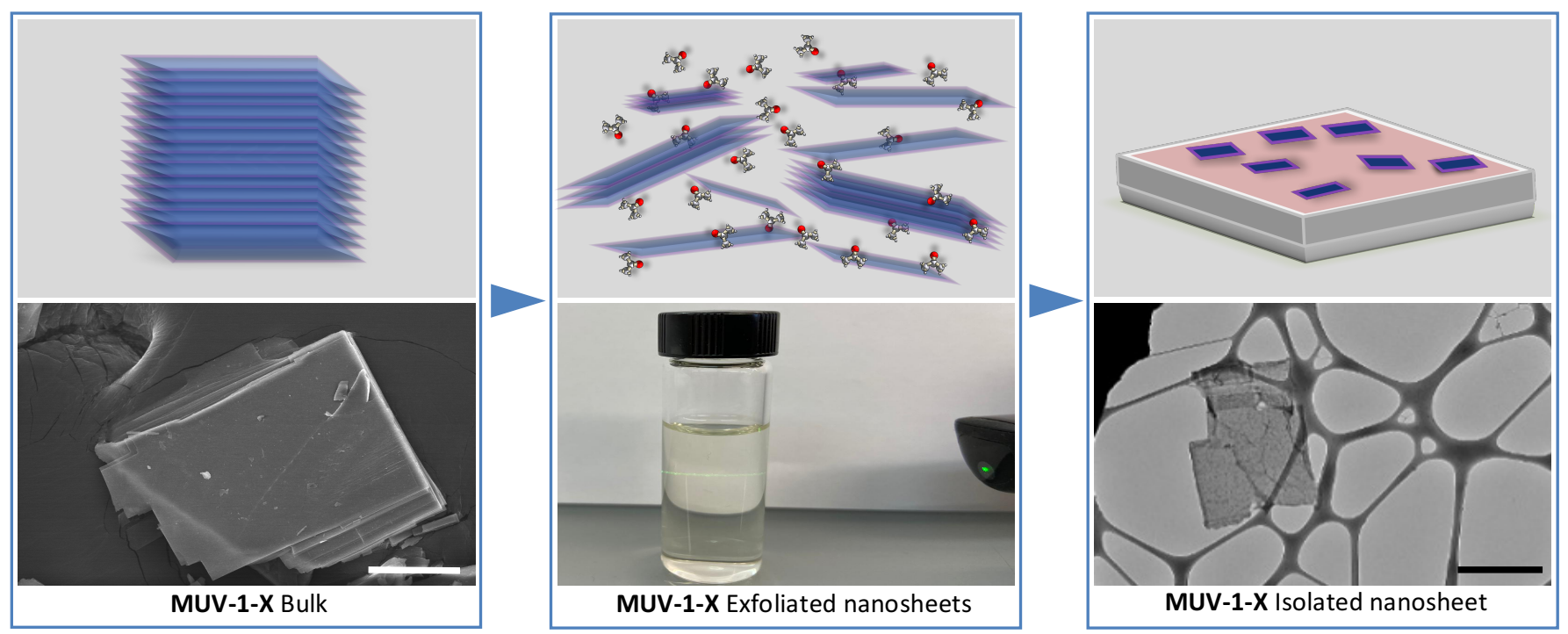

Figure 1. Exfoliation process from MUV-1-X (bulk) to isolated nanosheets. The top panels show a schematic representation of the process and the bottom panels show experimental images with MUV-1-Cl: (left) SEM image of a crystal of MUV-1-Cl (scale bar is $100 \mu \mathrm{m}$ ); (middle) Tindall effect of a suspension of delaminated MUV-1-Cl; (right) TEM image of a delaminated nanosheet of $\mathbf{M U V - 1 - C l}$ (scale bar is $2 \mu \mathrm{m}$ ).

successfully induces the delamination (see Figures S2 and S3). In fact, the damage to the flakes is much reduced when the sonication bath is applied at low temperature using an ice bath.

The different solvents that were used in order to study their relevance in the delamination process were selected due to their different properties: hydrogen bond donors, hydrogen bond acceptors and non-polar solvents. Specifically, we tested water, acetonitrile, diethyl ether, methanol, propanol, acetone, tetrahydrofurane, hexane, dichloromethane, and dimethylformamide. Delamination was successful with most of these solvents, with nanosheets of large lateral sizes observed with optical microscopy and Transmission Electronic Microscopy (TEM) (see Table S1 and Figure S4-S18). However, the behaviour of the exfoliated flakes differs with the solvent, with decomposition or agglomeration found in some cases in several hours (see Table S1), as shown by Raman spectroscopy and TEM (see Figures S4-S18). Only acetone and acetonitrile are found to be suitable solvents for a correct delamination, with higher stability and quality flakes observed for the former. In fact, exfoliated flakes are stable for several days in a colloidal suspension in acetone at low temperatures (see Figures S19 and S23).

After establishing the best solvent and source of energy to proceed with the delamination, we optimized the exposure time and concentration used in the exfoliation process. First, the delamination was analysed by changing the exposure time of the coordination polymer to sonication, resulting in a greater number of flakes with a large lateral size and small thickness in one hour. A reduced time of sonication causes a low delamination, resulting in very thick nanosheets, whereas a longer time of sonication causes the fragmentation of the nanosheets, resulting in very small lateral size (see Figures S24-S33). Second, we studied the effect of concentration on the exfoliation process, varying it from $0.0625 \mathrm{mg} / \mathrm{mL}$ to 0.75 $\mathrm{mg} / \mathrm{mL}$. An optimized concentration was $0.125 \mathrm{mg} / \mathrm{mL}$, with ca. $20 \%$ of the material successfully exfoliated; at lower concentration, practically no exfoliation was detected (see Figure S34). Finally, a study was carried out using different centrifugation conditions. This aspect was very important as it allowed us to achieve dispersions with homogeneous thicknesses (Figures S35-S42). The point at which the flakes were obtained with a more effective compromise between quantity and thickness is centrifuging at $8000 \mathrm{rpm}$, for 1 hour and maintaining a temperature of $5{ }^{\circ} \mathrm{C}$. The low temperature used in the centrifugation was shown to be critical, as also the storage temperature, which prevented the agglomeration and decomposition of the exfoliated material.

Using the above optimized conditions, we proceeded to an in-depth characterization of the exfoliated nanosheets of MUV-1-Cl. The
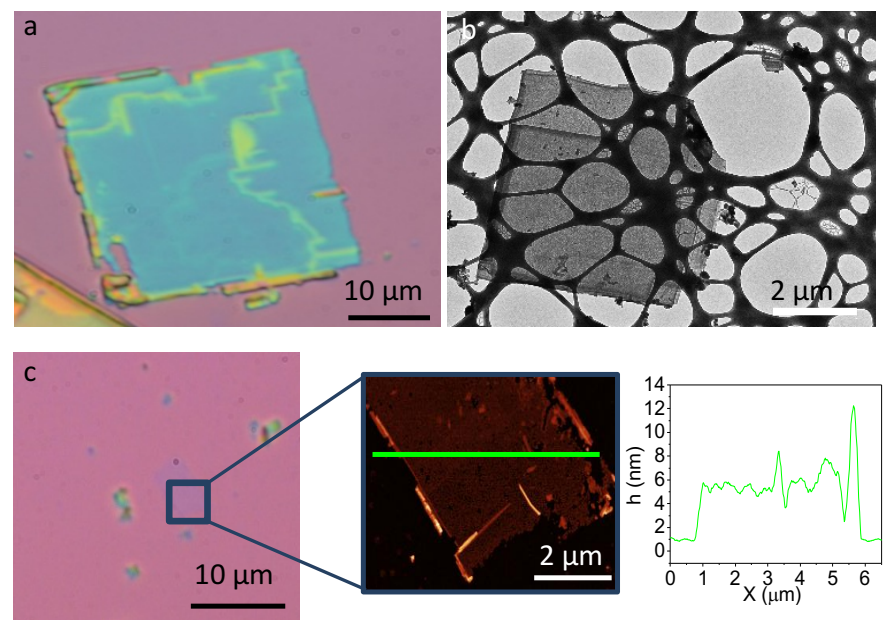

Figure 2. Characterization of MUV-1-Cl flakes achieved using the optimal conditions: sonication for one hour in a sonication bath, using acetone as a solvent and a concentration of $0.125 \mathrm{mg} / \mathrm{mL}$. (a) Optical image of a nanosheet with lateral sizes of $30 \times 35 \mu \mathrm{m}^{2}$. (b) TEM image of a few-layers flake ( $4 \mathrm{~nm}$ thickness). (c) Optical image with its corresponding AFM image and its height profile. 
isolation of the nanosheets were conducted by spin coating on $\mathrm{Si} / \mathrm{SiO}_{2}$ substrate a few drops of the top of the colloidal solution containing the exfoliated material, resulting in large amount of material (see Figures S43-S45). In this way flakes of different thicknesses and lateral sizes were isolated. Figure 2 shows flakes with lateral sizes much larger than previously reported, achieving values larger than $8 \mu \mathrm{m}$ (additional AFM images can be found in Figures S46S48 in the Supporting Information), with thicknesses as low as $4 \mathrm{~nm}$, corresponding to 3-4 layers. Although this is still far from the results obtained with purely inorganic materials, which are of the order of several microns of lateral size, ${ }^{24}$ exfoliated MUV-1-Cl flakes are much larger than other exfoliated 2D MOFs, with typical flakes of the order of 0.1-2 $\mu \mathrm{m}$ lateral sizes. ${ }^{21}$ Figure 3 shows the characterization of the exfoliated MUV-1-Cl flakes using Raman spectroscopy, AFM and Selected-Area Electron Diffraction (SAED). This multi-technique analysis allows the unequivocal demonstration of the chemical composition of the material, which has not been damaged after delamination. Quite remarkably, SAED reveals that the crystallinity of the material is maintained after liquid delamination.

In addition, the different members of the isoreticular MUV-1-X family, with $\mathrm{X}=\mathrm{CH}_{3}, \mathrm{Br}$, and $\mathrm{H}$, were successfully exfoliated following the same protocol as the established to delaminate MUV-1-Cl, achieving in all cases very similar results (Figure 3 and Figure S49S64). This indicates that the proposed methodology is valid for the different materials with different surface properties.

As far as the magnetism of these 2D MOFs is concerned, we noted that the magnetic signal of these flakes is too weak to be detected through conventional SQUID measurements. Still, a spectroscopic

a

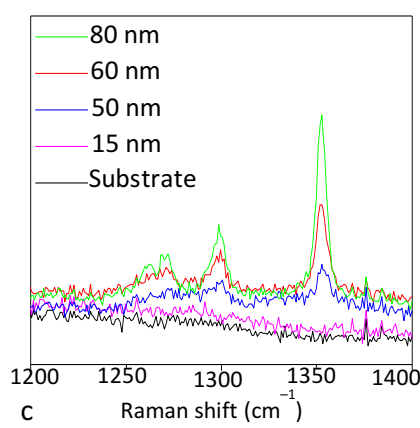

C

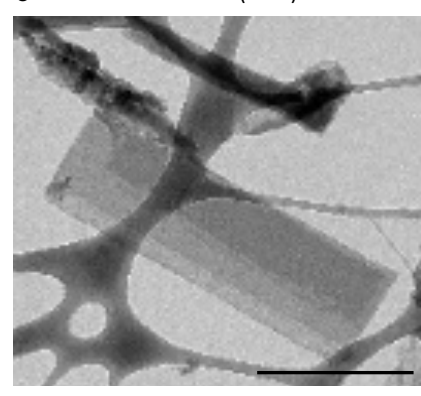

Figure 3. (a) Raman spectra of MUV-1-Cl flakes of different thicknesses; (b) AFM image and height profile of an exfoliated flake of MUV-1-Cl (scale bar = $2 \mu \mathrm{m}$ ); (c) Low-magnification TEM image of an exfoliated MUV-1-Cl nanosheet (scale bar = $1 \mu \mathrm{m}$ ); (d) the correspondent selected area electron diffraction pattern (SAED) (scale bar $=1 \mathrm{~nm}^{-1}$ ).

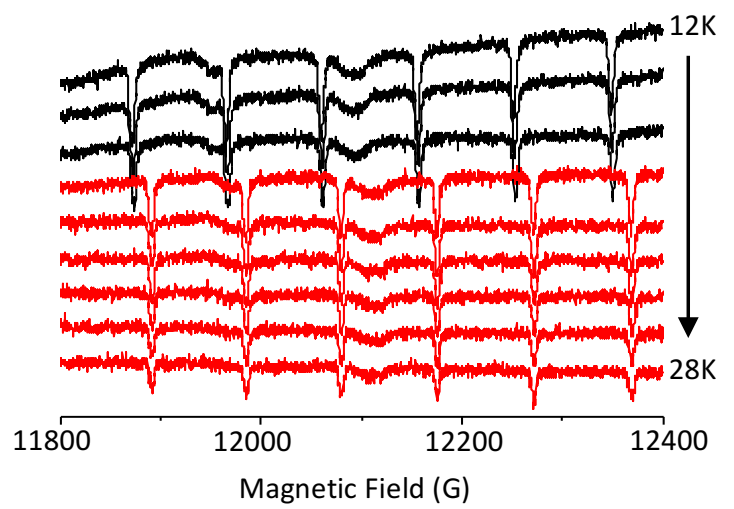

Figure 4. Electron paramagnetic resonance spectra of a dispersion of few layers MUV-1-Cl at different temperatures (every $2 \mathrm{~K}$ ), showing the sextet of $\mathrm{Mn}^{2+}$ (which is an impurity present in the solvent, see Figure S65 and S66). Upon magnetic ordering of the flakes, a clear shift of the signal towards lower fields is observed the exfoliated flakes preserve the magnetic ordering.

technique such as electron paramagnetic resonance (EPR) has shown to be very useful in this context. Thus, EPR of a suspension in acetone of few MUV-1-Cl flakes, which are intrinsically EPR silent, shows a sextet signal that can be assigned to a $\mathrm{Mn}^{2+}$ impurity, which is present in the acetone solvent (Figure 4 and Figure S66). This sextet corresponds to a hyperfine coupling constant of $96 \mathrm{G}(\mathrm{A}=270 \mathrm{MHz})$, compatible with the weak crystal field coming from oxygen-based $\mathrm{Mn}^{2+}$ complexes, such as $\left[\mathrm{Mn}\left(\mathrm{H}_{2} \mathrm{O}\right)_{6}\right]^{2+} \cdot{ }^{25}$ Interestingly, this $\mathrm{Mn}^{2+}$ sextet is shifted towards lower fields below a temperature of 16-18 $\mathrm{K}$, which is close to the ordering temperature of the bulk material (19-20 K). This strongly suggests that the ultrathin flakes retain the magnetic ordering of the bulk, showing small differences in the $T_{c}$ value that may be due to dimensionality effects, ${ }^{9}$ or simply to the distortion in the layers caused by the exfoliation process. The origin of this shift may be a consequence of the Zeeman splitting caused by the internal magnetic field created by the layers in the weak ferromagnetic state, which has to be added to the applied magnetic field. This requires close contact between the $\mathrm{Mn}^{2+}$ impurity and the sheets. We speculate that in the acetone suspension, $\mathrm{Mn}^{2+}$ is probably adsorbed on the surface of the sheets, or even coordinated to terminal benzimidazole ligands located at sheet defects. Similar magnetic shifts were previously observed in hybrid molecular magnets obtained upon insertion of paramagnetic metallocenium cations into layered oxalate magnets. ${ }^{26}$

In summary, this work shows the possibility of exfoliating the layered coordination polymers MUV-1-X using a liquid exfoliation method to obtain suspensions of magnetic 2D MOFs of high-quality, formed by layers with lateral sizes of several microns and very low thickness (down to $4 \mathrm{~nm}$ ). Interestingly, these magnetic nanosheets maintain their structural integrity and the magnetic ordering. This study paves the way for the use of these metal-organic nanosheets in functional heterostructures via a chemical approach which is unachievable by dry exfoliation. 


\section{Conflicts of interest}

There are no conflicts to declare

\section{Acknowledgements}

We acknowledge funding by the EU (ERC Consolidator Grant SCAGE (724681) and ERC Advanced Grant 2D-MOL (788222), the Spanish MINECO (projects MAT2017-89993-R and CTQ201789528-P cofinanced by FEDER and Excellence Unit María de Maeztu MDM-2015-0538), and the Generalitat Valenciana (PROMETEO Program). G.M.E. acknowledges funding by the MINECO (Ramón y Cajal Program). J.L.-C. acknowledges the Universitat de Valencia for an "Atracció de Talent" grant. Financial support from the Generalitat Valenciana (PO FEDER, ref. IDIFEDER/2018/061) is also acknowledged. We also acknowledge J. M. Martínez-Agudo and G. Agustí for their help with the EPR measurements, and A. Soriano-Portillo for her help in AFM measurements.

\section{Notes and references}

1 C. Tan, X. Cao, X. J. Wu, Q. He, J. Yang, X. Zhang, J. Chen, W. Zhao, S. Han, G. H. Nam, M. Sindoro and H. Zhang, Chem. Rev., 2017, 117, 6225-6331.

2 A. C. Ferrari, et al. Nanoscale, 2015, 7, 4598-4810.

3 (a) D. Rodríguez-San-Miguel, P. Amo-Ochoa and F. Zamora, Chem. Commun., 2016, 52, 4113-4127. (b) D. J. Ashworth and J. A. Foster, J. Mater. Chem. A, 2018, 6, 16292-16307. (c) M. A. Solomos, F. J. Claire and T. J. Kempa, J. Mater. Chem. A, 2019, 7, 23537-23562.(d) J. Duan, Y. Li, Y. Pan, N. Behera and W. Jin, Coord. Chem. Rev., 2019, 395, 25-45. (e) W. Liu, R. Yin, X. Xu, L. Zhang, W. Shi and X. Cao, Adv. Sci., 2019, 6, 1892373. (f) Y. Z. Li, Z. H. Fu and G. Xu, Coord. Chem. Rev., 2019, 388, 79-106. (g) R. Sakamoto, K. Takada, T. Pal, H. Maeda, T. Kambe and H. Nishihara, Chem. Commun., 2017, 53, 5781-5801. (h) R. Dong, T. Zhang and X. Feng, Chem. Rev., 2018, 118, 6189-6325.

4 A. Dhakshinamoorthy, A. M. Asiri and H. Garcia, Adv. Mater., 2019, 31, 1900617.

5 (a) H. Zhu and D. Liu, J. Mater. Chem. A, 2019, 7, 21004-21035. (b) C. Zhang, B. H. Wu, M. Q. Ma, Z. Wang and Z. K. Xu, Chem. Soc. Rev., 2019, 48, 3811-3841.

6 (a) S. Zhao, Y. Wang, J. Dong, C. He, H. Yin, P. An, Kun Zhao, X. Zhang, C. Gao, L. Zhang, J. Lv, J. Wang, J. Zhang, A. Muqsit Khattak, N. Ali Khan, Z. Wei, Jing Zhang, S. Liu, H. Zhao and Z. Tang Nat. Energy, 2016, 1, 16184. (b) Z. Wang, G. Wang, H. Qi, M. Wang, M. Wang, S. Park, H. Wang, M. Yu, U. Kaiser, A. Fery, S. Zhou, R. Dong and X. Feng, Chem. Sci., 2020, DOI:10.1039/D0SC01408G.

7 (a) M. Ko, L. Mendecki and K. A. Mirica, Chem. Commun., 2018, 54, 78737891.(b) R. Dong, et al. Nat. Mater., 2018, 17, 1027-1032. (c) C. Yang, et al. Nat. Commun., 2019, 10, 3260. (d) V. Rubio-Giménez, S. Tatay, F. Volatron, F. J. Martínez-Casado, C. Martí-Gastaldo and E. Coronado, J. Am. Chem. Soc., 2016, 138, 2576-2584. (e) V. Rubio-Giménez, et al. Adv. Mater., 2018, 30, 170429.

8 E. Coronado, Nat. Rev. Mater., 2020, 5, 87-104.

9 B. Huang et al. Nature, 2017, 546, 270-273.

10 A. Abhervé, S. Mañas-Valero, M. Clemente-León and E. Coronado, Chem. Science, 2015, 6, 4665-4673.

11 J. López-Cabrelles, et al. Nat. Chem., 2018, 10, 1001-1007.

12 (a) K. S. Pedersen, et al. Nat. Chem., 2018, 10, 1056-1061. (b) S. SuarezGarcia, et al. ACS Appl. Nano Mater., 2018, 1, 2662-2668. (c) S. Benmansour, A. Abhervé, P. Gómez-Claramunt, C. Vallés-García and C. J. Gómez-García, ACS Appl. Mater. Interfaces, 2017, 9, 26210-26218. (d) P. J. Saines, M. Steinmann, J. C. Tan, H. H. M. Yeung, W. Li, P. T. Barton and A. K. Cheetham, Inorg. Chem., 2012, 51, 11198-11209. (e) Q. Tang, Z. Zhou and Z. Chen, J. Phys. Chem. C, 2012, 116, 4119-4125. (f) A. Stoddart, Nat.
Rev. Mater., 2018, 3, 357.

13 Y. Peng and W. Yang, Adv. Mater. Interfaces, 2020, 7, 1901514.

14 (a) T. Rodenas, I. Luz, G. Prieto, B. Seoane, H. Miro, A. Corma, F. Kapteijn, F. X. Llabrés I Xamena and J. Gascon, Nat. Mater., 2015, 14, 48-55. (b) C. Li, L. Shi, L. Zhang, P. Chen, J. Zhu, X. Wang and Y. Fu, J. Mater. Chem. A, 2019, 8, 369-379. (c) L. Zhuang, et al. Angew. Chemie - Int. Ed., 2019, 58, 13565-13572.(d) M. Jian, H. Liu, T. Williams, J. Ma, H. Wang and X. Zhang, Chem. Commun., 2017, 53, 13161-13164.(e) L. Cao, Z. Lin, F. Peng, W. Wang, R. Huang, C. Wang, J. Yan, J. Liang, Z. Zhang, T. Zhang, L. Long, J. Sun and W. Lin, Angew. Chemie - Int. Ed., 2016, 55, 4962-4966. (f) Y. N. Li, S. Wang, Y. Zhou, X. J. Bai, G. S. Song, X. Y. Zhao, T. Q. Wang, X. Qi, X. M. Zhang and Y. Fu, Langmuir, 2017, 33, 1060-1065. (g) A. Pustovarenko, et al. Adv. Mater., 2018, 30, 1707234.

15 E. Gao, S. Z. Lin, Z. Qin, M. J. Buehler, X. Q. Feng and Z. Xu, J. Mech. Phys. Solids, 2018, 115, 248-262.

16 V. Nicolosi, M. Chhowalla, M. G. Kanatzidis, M. S. Strano and J. N. Coleman, Science, 2013, 340, 1226419.

17 (a) Y. Cao, V. Fatemi, S. Fang, K. Watanabe, T. Taniguchi, E. Kaxiras and P. Jarillo-Herrero. Nature, 2018, 556, 43-50. (b) D. R. Klein, et al., Nat. Phys., 2019, 15, 1255-12.

18 Y. Peng, Y. Li, Y. Ban, H. Jin, W. Jiao, X. Liu and W. Yang, Science. 2014, 346, 1356-1359.

19 D. J. Ashworth, A. Cooper, M. Trueman, R. W. M. Al-Saedi, L. D. Smith, A. J. H. M. Meijer and J. A. Foster, Chem. - A Eur. J., 2018, 24, 17986-17996.

20 C. Tan, K. Yang, J. Dong, Y. Liu, Y. Liu, J. Jiang and Y. Cui, J. Am. Chem. Soc, 2019, 141, 17685-17695.

21 (a) V. K. Maka, A. Mukhopadhyay, S. Jindal and J. N. Moorthy, Chem. - A Eur. J., 2019, 25, 3835-3842. (b) K. Jayaramulu, J. Masa, D. M. Morales, O. Tomanec, V. Ranc, M. Petr, P. Wilde, Y. T. Chen, R. Zboril, W. Schuhmann and R. A. Fischer, Adv. Sci., 2018, 5, 1801029. (c) W. M. Liao, et al. Nat. Commun., 2018, 9, 2401. (d) J. A. Foster, S. Henke, A. Schneemann, R. A. Fischer and A. K. Cheetham, Chem. Commun., 2016, 52, 10474-10477. (e) V. K. M. Au, K. Nakayashiki, H. Huang, S. Suginome, H. Sato and T. Aida, J. Am. Chem. Soc., 2019, 141, 53-57. (f) J. X. Wu, W. W. Yuan, M. Xu and Z. Y. Gu, Chem. Commun., 2019, 55, 11634-11637. (g) Y. H. Luo, C. Chen, C. He, Y. Y. Zhu, D. L. Hong, X. T. He, P. J. An, H. S. Wu and B. W. Sun, ACS Appl. Mater. Interfaces, 2018, 10, 28860-28867. (h) C. Hermosa, B. R. Horrocks, J. I. Martínez, F. Liscio, J. Gómez-Herrero and F. Zamora, Chem. Sci., 2015, 6, 25532558. (i) A. Kondo, C. C. Tiew, F. Moriguchi and K. Maeda, Dalt. Trans., 2013, 42, 15267-15270. (j) T. Araki, A. Kondo and K. Maeda, Chem. Commun., 2013, 49, 552-554. (k) P. Amo-Ochoa, L. Welte, R. González-Prieto, P. J. Sanz Miguel, C. J. Gómez-García, E. MateoMartí, S. Delgado, J. Gómez-Herrero and F. Zamora, Chem. Commun., 2010, 46, 3262. (I) D. Liu, B. Liu, C. Wang, W. Jin, Q. Zha, G. Shi, D. Wang, X. Sang and C. Ni, ACS Sustain. Chem. Eng., 2020, 8, 2167-2175.

22 N. Contreras-Pereda, P. Hayati, S. Suárez-García, L. Esrafili, P. Retailleau, S. Benmansour, F. Novio, A. Morsali and D. Ruiz-Molina, Ultrason. Sonochem., 2019, 55, 186-195.

23 (a) J. López-Cabrelles, J. Romero, G. Abellán, M. Giménez-Marqués, M. Palomino, S. Valencia, F. Rey and G. Mínguez Espallargas, J. Am. Chem. Soc., 2019, 141, 7173-7180. (b) J. López-Cabrelles, M. Giménez-Marqués, G. Mínguez Espallargas and E. Coronado, Inorg. Chem., 2015, 54, 1049010496.

24 (a) C. Gibaja, et al. Angew. Chemie - Int. Ed., 2016, 55, 14345-14349. (b) D. Hanlon, et al. Nat. Commun., 2015, 6, 8563. (c) M. Lotya, P. J. King, U. Khan, S. De and J. N. Coleman, ACS Nano, 2010, 4, 3155-3162. (d) A. O'Neill, U. Khan and J. N. Coleman, Chem. Mater., 2012, 24, 2414-2421. (e) C. Backes, T. M. Higgins, A. Kelly, C. Boland, A. Harvey, D. Hanlon and J. N. Coleman, Chem. Mater., 2017, 29, 243-255.

25 (a) N. Cox, W. Lubitz and A. Savitsky, Mol. Phys., 2013, 111, 2788-2808. (b) K. Keller, M. Zalibera, M. Qi, V. Koch, J. Wegner, H. Hintz, A. Godt, G. Jeschke, A. Savitsky and M. Yulikov, Phys. Chem. Chem. Phys., 2016, 18, 25120-25135.

26 E. Coronado, J. Galán-Mascarós, C. Gómez-García, J. Ensling and P. Gütlich, Chem. A Eur. J., 2000, 6, 552-563. 